\title{
Fuzzy stability of a cubic functional equation via fixed point technique
}

\author{
Syed Abdul Mohiuddine* and Abdullah Alotaibi
}

* Correspondence: mohiuddine@gmail.com Department of Mathematics,

Faculty of Science, King Abdulaziz University, P.O. Box 80203, Jeddah

21589, Saudi Arabia

\section{Abstract \\ The object of this article is to determine Hyers-Ulam-Rassias stability results concerning the cubic functional equation in fuzzy normed space by using the fixed point method.}

Keywords: Hyers-Ulam-Rassias stability, cubic functional equation, fuzzy normed space, fixed point

\section{Introduction, definitions and notations}

Fuzzy set theory is a powerful hand set for modeling uncertainty and vagueness in various problems arising in the field of science and engineering. It has a large number of application, for instance, in the computer programming [1], engineering problems [2], statistical convergence [3-7], nonlinear operator [8], best approximation [9] etc. Particularly, fuzzy differential equation is a strong topic with large application areas, for example, in population models [10], civil engineering [11] and so on.

By modifying own studies on fuzzy topological vector spaces, Katsaras [12] first introduced the notion of fuzzy seminorm and norm on a vector space and later on Felbin [13] gave the concept of a fuzzy normed space (for short, FNS) by applying the notion fuzzy distance of Kaleva and Seikala [14] on vector spaces. Further, Xiao and Zhu [15] improved a bit the Felbin's definition of fuzzy norm of a linear operator between FNSs.

Stability problem of a functional equation was first posed by Ulam [16] which was answered by Hyers [17] under the assumption that the groups are Banach spaces. Rassias [18] and Gajda [19] considered the stability problem with unbounded Cauchy differences. The unified form of the results of Hyers, Rassias, and Gajda is as follows:

Let $E$ and $F$ be real normed spaces with $F$ complete and let $f: E \rightarrow F$ be a mapping such that the following condition holds

$$
\|f(x+y)-f(x)-f(y)\|_{F} \leq \theta\left(\|x\|_{E}^{p}+\|y\|_{E}^{p}\right),
$$

for all $x, y \in E, \theta \geq 0$ and for some $p \in[0, \infty) \mid\{1\}$. Then there exists a unique additive function $C: E \rightarrow F$ such that

$$
\|f(x)-C(x)\|_{F} \leq \frac{2 \theta}{\left|2-2^{p}\right|}\|x\|_{E}^{p}
$$

(C) 2012 Mohiuddine and Alotaibi; licensee Springer. This is an open access article distributed under the terms of the Creative Commons Attribution License (http://creativecommons.org/licenses/by/2.0), which permits unrestricted use, distribution, and reproduction in any medium, provided the original work is properly cited. 
for all $x \in E$.

This stability phenomenon is called generalized Hyers-Ulam stability and has been extensively investigated for different functional equations. It is to be noted that almost all proofs used the idea imaginated by Hyers. Namely, the additive function $C: E \rightarrow F$ is explicitly constructed, starting from the given function $f$, by the formulae (i) $C(x)=\lim _{n \rightarrow \infty} \frac{1}{2^{n}} f\left(2^{n} x\right)$, if $p<1$; and (ii) $C(x)=\lim _{n \rightarrow \infty} 2^{n} f\left(\frac{x}{2^{n}}\right)$, if $p>1$. This method is called a direct method. It is often used to construct a solution of a given functional equation and is seen to be a powerful tool for studying the stability of many functional equations. Since then several stability problems and its fuzzy version for various functional equations have been investigated in [20-26]. Recently, Radu [27] proposed that fixed point alternative method is very useful for obtaining the solution of Ulam problem.

The stability problem for the cubic functional equation was proved by Jun and Kim [21] for mappings $f: X \rightarrow Y$, where $X$ is a real normed space and $Y$ is a Banach space. In this article, we show that the existence of the limit $C(x)$ and the estimation (i) and (ii) can be simply obtained from the alternative of fixed point.

In this section, we recall some notations and basic definitions used in this article.

A fuzzy subset $\mathrm{N}$ of $X \times \mathbb{R}$ is called a fuzzy norm on $X$ if the following conditions are satisfied for all $x, y \in X$ and $c \in \mathbb{R}$;

(a) $N(x, t)=0$ for all non-positive $t \in \mathbb{R}$,

(b) $N(x, t)=1$ for all $t \in \mathbb{R}^{+}$if and only if $x=0$,

(c) $N(c x, t)=N\left(x, \frac{t}{|c|}\right)$ for all $t \in \mathbb{R}^{+}$and $c \neq 0$,

(d) $N(x+y, t+s) \geq \min \{N(x, t), N(y, s)\}$ for all $s, t \in \mathbb{R}$,

(e) $N(x, t)$ is a non-decreasing function on $\mathbb{R}$, and $\sup _{t \in \mathbb{R}} N(x, t)=1$.

The pair $(X, N)$ will be referred to as a fuzzy normed space.

Example 1.1. Let $(X,\|\|$.$) be a normed linear space. Then$

$$
N(x, t)= \begin{cases}\frac{t}{t+\|x\|} & \text { if } t>0 \\ 0 & \text { if } t \leq 0,\end{cases}
$$

is a fuzzy norm on $X$.

Example 1.2. Let $(X,\|\|$.$) be a normed linear space. Then$

$$
N(x, t)=\left\{\begin{array}{l}
1 \text { if } t>\|x\|, \\
0 \text { if } t \leq\|x\|,
\end{array}\right.
$$

is a fuzzy norm on $X$.

Let $(X, N)$ be a fuzzy normed space. Then, a sequence $x=\left(x_{k}\right)$ is said to be fuzzy convergent to $L \in X$ if $\lim N\left(x_{k}-L, t\right)=1$, for all $t>0$. In this case, we write $N$-lim $x_{k}=L$.

Let $(X, N)$ be an fuzzy normed space. Then, $x=\left(x_{k}\right)$ is said to be fuzzy Cauchy sequence if $\lim N\left(x_{k+p}-x_{k}, t\right)=1$ for all $t>0$ and $p=1,2, \ldots$.

It is known that every convergent sequence in a fuzzy normed space $(X, N)$ is Cauchy. Fuzzy normed space $(X, N)$ is said to be complete if every fuzzy Cauchy sequence is fuzzy convergent. In this case, $(X, N)$ is called fuzzy Banach space. 


\section{Fixed point technique for Hyers-Ulam stability}

In this section, we deal with the stability problem via fixed point method in fuzzy norm space. Before proceeding further, we should recall the following results related to the concept of fixed point.

Theorem 2.1 (Banach's Contraction principle). Let $(X, d)$ be a complete generalized metric space and consider a mapping $J: X \rightarrow X$ be a strictly contractive mapping, that is

$$
d(J x, J y) \leq L d(x, y), \forall x, y \in X
$$

for some (Lipschitz constant) $L<1$. Then

(i) The mapping $J$ has one and only one fixed point $x^{*}=J\left(x^{*}\right)$;

(ii) The fixed point $x^{*}$ is globally attractive, that is

$$
\lim _{n \rightarrow \infty} J^{n} x=x^{*}
$$

for any starting point $x \in X$;

(iii) One has the following estimation inequalities for all $x \in X$ and $n \geq 0$ :

$$
\begin{aligned}
& d\left(J^{n} x, x^{*}\right) \leq L^{n} d\left(x, x^{*}\right) \\
& d\left(J^{n} x, x^{*}\right) \leq \frac{1}{1-L} d\left(J^{n} x, J^{n+1} x\right) \\
& d\left(x, x^{*}\right) \leq \frac{1}{1-L} d(x, J x) .
\end{aligned}
$$

Theorem 2.2 (The alternative of fixed point) [28]. Suppose we are given a complete generalized metric space $(X, d)$ and a strictly contractive mapping $J: X \rightarrow X$, with Lipschitz constant $L$. Then, for each given element $x \in X$, either

$$
d\left(J^{n} x, J^{n+1} x\right)=+\infty, \forall n \geq 0
$$

or

$$
d\left(J^{n} x, J^{n+1} x\right)<+\infty \forall n \geq n \text { 。 }
$$

for some natural number $n_{0}$. Moreover, if the second alternative holds then

(i) The sequence $\left(J^{n} x\right)$ is convergent to a fixed point $y^{*}$ of $J$;

(ii) $y^{*}$ is the unique fixed point of $J$ in the set $Y=\left\{y \in X, d\left(J^{n_{\circ}} x, y\right)<+\infty\right\}$

(iii) $d\left(y, y^{*}\right) \leq \frac{1}{1-L} d(y, J y), y \in Y$.

We are now ready to obtain our main results.

The functional equation

$$
f(2 x+y)+f(2 x-y)=2 f(x+y)+2 f(x-y)+12 f(x)
$$


is called the cubic functional equation, since the function $f(x)=c x^{3}$ is its solution. Every solution of the cubic functional equation is said to be a cubic mapping.

Let $\phi$ be a function from $X \times X$ to $Z$. A mapping $f: X \rightarrow Y$ is said to be $\phi$-approximately cubic function if

$$
N(f(2 x+y)+f(2 x-y)-2 f(x+y)-2 f(x-y)-12 f(x), t) \geq N^{\prime}(\varphi(x, y), t)
$$

for all $x, y \in X$ and $t>0$.

Using the fixed point alternative, we can prove the stability of Hyers-Ulam-Rassias type theorem in FNS. First, we prove the following lemma which will be used in our main result.

Lemma 2.1. Let $\left(Z, N^{\prime}\right)$ be a fuzzy normed space and $\phi: X \rightarrow Z$ be a function. Let $E$ $=\{g: X \rightarrow Y ; g(0)=0\}$ and define

$$
d_{M}(g, h)=\inf \left\{a \in \mathbb{R}^{+}: N(g(x)-h(x), a t) \geq N^{\prime}(\varphi(x, 0), t) \text { for all } x \in X \text { and } t>0\right\},
$$

for all $h L E$. Then $d_{M}$ is a complete generalized metric on $E$.

Proof. Let $g, h, k L E, d_{M}(g, h)<\xi_{1}$ and $d_{M}(h, k)<\xi_{2}$. Then

$$
N\left(g(x)-h(x), \xi_{1} t\right) \geq N^{\prime}(\varphi(x), t) \text { and } N\left(h(x)-k(x), \xi_{2} t\right) \geq N^{\prime}(\varphi(x), t),
$$

for all $x L X$ and $t>0$. Thus

$$
N\left(g(x)-k(x),\left(\xi_{1}+\xi_{2}\right) t\right) \geq \min \left\{N\left(g(x)-h(x), \xi_{1} t\right), N\left(h(x)-k(x), \xi_{2} t\right)\right\} \geq N^{\prime}(\varphi(x), t),
$$

for each $x L X$ and $t>0$. By definition $d_{M}(h, k)<\xi_{1}+\xi_{2}$. This proves the triangle inequality for $d_{M}$. Rest of the proof can be done on the same lines as in (see [[29], Lemma 2.1]).

Theorem 2.3. Let $X$ be a linear space and $\left(Z, N^{\prime}\right)$ be a FNS. Suppose that a function $\phi: X \times X \rightarrow Z$ satisfying $\phi(2 x, 2 y)=\alpha \phi(x, y)$ for all $x, y L X$ and $\alpha \neq 0$. Suppose that $(Y, N)$ be a fuzzy Banach space and $f: X \rightarrow Y$ be a $\phi$-approximately cubic function. If for some $0<\alpha<8$

$$
N^{\prime}(\varphi(2 x, 0), t) \geq N^{\prime}(\alpha \varphi(x, 0), t),
$$

and

$$
\lim _{n \rightarrow \infty} N^{\prime}\left(\varphi\left(2^{n} x, 2^{n} \gamma\right), 8^{n} t\right)=1,
$$

for all $x, y \in X$ and $t>0$. Then there exists a unique cubic mapping $C: X \rightarrow Y$ such that

$$
N(C(x)-f(x), t) \geq N^{\prime}(\varphi(x, 0), 2(8-\alpha) t),
$$

for all $x \in X$ and all $t>0$.

Proof. Put $y=0$ in (2.0.2). Then for all $x \in X$ and $t>0$

$$
N\left(\frac{f(2 x)}{8}-f(x), \frac{t}{16}\right) \geq N^{\prime}(\varphi(x, 0), t) .
$$

Consider the set $E=\{g: X \rightarrow Y, g(0)=0\}$ together with the mapping $d_{M}$ defined on $E \times E$ by

$$
d_{M}(g, h)=\inf \left\{a \in \mathbb{R}^{+}: N(g(x)-h(x), a t) \geq N^{\prime}(\varphi(x, 0), t) \text { for all } x \in X \text { and } t>0\right\} .
$$


It is known that $d_{M}(g, h)$ complete generalized metric space by Lemma 2.1. Now, we define the linear mapping $J: E \rightarrow E$ such that

$$
J g(x)=\frac{1}{8} g(2 x)
$$

It is easy to see that $J$ is a strictly contractive self-mapping of $E$ with the Lipschitz constant $\frac{\alpha}{8}$. Indeed, let $g, h L E$ be given such that $d_{M}(g, h)=\varepsilon$. Then

$$
N(g(x)-h(x), \varepsilon t) \geq N^{\prime}(\varphi(x, 0), t),
$$

for all $x \in X$ and $t>0$. Thus

$$
\begin{aligned}
N\left(J g(x)=J h(x), \frac{\alpha}{8} \varepsilon t\right) & =N\left(\frac{1}{8} g(2 x)-\frac{1}{8} h(2 x), \frac{\alpha}{8} \varepsilon t\right) \\
& =N(g(2 x)-h(2 x), \alpha \varepsilon t) \geq N^{\prime}(\varphi(2 x, 0), \alpha t) .
\end{aligned}
$$

It follows from (2.3.1) that

$$
N\left(J g(x)-J h(x), \frac{\alpha}{8} \varepsilon t\right) \geq N^{\prime}(\alpha \varphi(x, 0), \alpha t)=N^{\prime}(\varphi(x, 0), t),
$$

for all $x \in X$ and $t>0$. Therefore

$$
d_{M}(g, h)=\varepsilon \Rightarrow d_{M}(J g, J h) \leq \frac{\alpha}{8} \varepsilon .
$$

This means that

$$
d_{M}(J g, J h) \leq \frac{\alpha}{8} d_{M}(g, h),
$$

for all $g, h \in E$. Next, from

$$
N\left(\frac{f(2 x)}{8}-f(x), \frac{t}{16}\right) \geq N^{\prime}(\varphi(x, 0), t),
$$

we have $d_{M}(f, J f) \leq \frac{1}{16}$

Using the fixed point alternative we deduce the existence of a fixed point of $J$, that is, the existence of a mapping $C: X \rightarrow Y$ such that $C(2 x)=8 C(x)$, for all $x L X$. Moreover, we have $d_{M}\left(J^{n} f, C\right) \rightarrow 0$, which implies

$$
N-\lim _{n} \frac{f\left(2^{n} x\right)}{8^{n}}=C(x),
$$

for every $x L X$. Also

$$
d_{M}(f, C) \leq \frac{1}{1-L} d_{M}(f, J f) \text { implies } d_{M}(f, C) \leq \frac{1}{16\left(1-\frac{\alpha}{8}\right)}=\frac{1}{2(8-\alpha)} .
$$

This implies that

$$
N\left(C(x)-f(x), \frac{1}{2(8-\alpha)} t\right) \geq N^{\prime}(\varphi(x, 0), t) .
$$


Replacing $t$ by $2(8-\alpha) t$ in the above equation, we obtain

$$
N(C(x)-f(x), t) \geq N^{\prime}(\varphi(x, 0), 2(8-\alpha) t),
$$

for all $x L X$ and $t>0$.

Let $x, y\lfloor X$. Then

$$
N(C(2 x+y)+C(2 x-y)-2 C(x+y)-2 C(x-y)-12 C(x), t) \geq N^{\prime}(\varphi(x, y), t) .
$$

Replacing $x$ and $y$ by $2^{n} x$ and $2^{n} y$, respectively, we obtain

$$
\begin{aligned}
& N\left(\frac{C\left(2^{n}(2 x+y)\right)}{8^{n}}+\frac{C\left(2^{n}(2 x-y)\right)}{8^{n}}-\frac{2 C\left(2^{n}(x+y)\right)}{8^{n}}-\frac{2 C\left(2^{n}(x-y)\right)}{8^{n}}-\frac{12 C\left(2^{n} x\right)}{8^{n}}, t\right) \\
& \quad \geq N^{\prime}\left(\varphi\left(2^{n} x, 2^{n} y\right), 8^{n} t\right)
\end{aligned}
$$

for all $x, y\lfloor X$ and all $t>0$. Since

$$
\lim _{n \rightarrow \infty} N^{\prime}\left(\varphi\left(2^{n} x, 2^{n} y\right), 8^{n} t\right)=1,
$$

we conclude that $C$ fulfills (2.0.1).

The uniqueness of $C$ follows from the fact that $C$ is the unique fixed point of $J$ with the following property that there exists $u L(0, \infty)$ such that

$$
N(C(x)-f(x), u t) \geq N^{\prime}(\varphi(x, 0), t),
$$

for all $x L X$ and $t>0$.

This completes the proof of the theorem.

By a modification in the proof of Theorem 2.3, one can prove the following:

Theorem 2.4. Let $X$ be a linear space and $\left(Z, N^{\prime}\right)$ be a FNS. Suppose that a function $\phi: X \times X \rightarrow Z$ satisfying

$$
\varphi\left(\frac{x}{2}, \frac{y}{2}\right)=\frac{1}{\alpha} \varphi(x, y)
$$

for all $x, y\lfloor X$ and $\alpha \neq 0$. Suppose that $(Y, N)$ be a fuzzy Banach space and $f: X \leftarrow Y$ be a $\phi$-approximately cubic function. If for some $\alpha>8$

$$
N^{\prime}(\varphi(x / 2,0), t) \geq N^{\prime}(\varphi(x, 0), \alpha t),
$$

and

$$
\lim _{n \rightarrow \infty} N^{\prime}\left(8^{-n} \varphi\left(2^{-n} x, 2^{-n} y\right), t\right)=1
$$

for all $x, y L X$ and $t>0$. Then there exists a unique cubic mapping $C: X \rightarrow Y$ such that

$$
N(C(x)-f(x), t) \geq N^{\prime}(\varphi(x, 0), 2(\alpha-8) t),
$$

for all $x L X$ and all $t>0$.

The proof of the above theorem is similar to the proof of Theorem 2.3, hence omitted.

\section{Conclusion}

This study indeed presents a relationship between three various disciplines: the theory of fuzzy normed spaces, the theory of stability of functional equations and the fixed point theory. This method is easier than those of previously proved (stability problem) 
by other authors for fuzzy setting. We established Hyers-Ulam-Rassias stability of a cubic functional equation in fuzzy normed spaces by using fixed point alternative theorem.

\section{Author' information}

Address of both the authors: Department of Mathematics, Faculty of Science, King Abdu-laziz University, P.O. Box 80203, Jeddah 21589, Saudi Arabia. Email: mohiuddine@gmail.com (S.A. Mohiuddine); mathker11@hotmail.com (A. Alotaibi).

\section{Acknowledgements}

The authors would like to thank the anonymous reviewers for their valuable comments.

\section{Authors' contributions}

Both the authors contributed equally and significantly in writing this paper. All authors read and approved the final manuscript.

\section{Competing interests}

The authors declare that they have no competing interests.

Received: 20 January 2012 Accepted: 17 April 2012 Published: 17 April 2012

\section{References}

1. Giles, R: A computer program for fuzzy reasoning. Fuzzy Sets Syst. 4, 221-234 (1980)

2. Hanss, M: Applied fuzzy arithmetic: an introduction with engineering applications. Springer-Verlag, Berlin (2005)

3. Mohiuddine, SA, Danish Lohani, QM: On generalized statistical convergence in intuitionistic fuzzy normed space. Chaos Solitons Fract. 42, 1731-1737 (2009)

4. Mursaleen, M, Mohiuddine, SA: Statistical convergence of double sequences in intuitionistic fuzzy normed spaces. Chaos Solitons Fract. 41, 2414-2421 (2009)

5. Mursaleen, M, Mohiuddine, SA: On lacunary statistical convergence with respect to the intuitionistic fuzzy normed space. J Comput Appl Math. 233(2):142-149 (2009)

6. Mursaleen, M, Mohiuddine, SA, Edely, OHH: On the ideal convergence of double sequences in intuitionistic fuzzy normed spaces. Comput Math Appl. 59, 603-611 (2010)

7. Mohiuddine, SA, Şevli, H, Cancan, M: Statistical convergence in fuzzy 2-normed space. J Computational Analy Appl. 12(4):787-798 (2010)

8. Mursaleen, M, Mohiuddine, SA: Nonlinear operators between intuitionistic fuzzy normed spaces and Fr'echet differentiation. Chaos Solitons Fract. 42, 1010-1015 (2009)

9. Mohiuddine, SA: Some new results on approximation in fuzzy 2-normed spaces. Math Comput Model. 53, 574-580 (2011)

10. Guo, M, Li, R: Impulsive functional differential inclusions and fuzzy population models. Fuzzy Sets Syst. 138, 601-615 (2003)

11. Oberguggenberger, M, Pittschmann, S: Differential equations with fuzzy parameters. Math Mod Syst. 5, 181-202 (1999)

12. Katsaras, AK: Fuzzy topological vector spaces. Fuzzy Sets Syst. 12, 143-154 (1984)

13. Felbin, C: Finite dimensional fuzzy normed linear spaces. Fuzzy Sets Syst. 48, 239-248 (1992)

14. Kaleva, O, Seikala, S: On fuzzy metric spaces. Fuzzy Sets Syst. 12, 215-229 (1984)

15. Xiao, JZ, Zhu, XH: Fuzzy normed spaces of operators and its completeness. Fuzzy Sets Syst. 133(3):135-146 (2003)

16. Ulam, SM: A Collection of the Mathematical Problems. Interscience Publ. New York (1960)

17. Hyers, DH: On the stability of the linear functional equation. Proc Natl Acad Sci USA. 27, $222-224$ (1941)

18. Rassias, TM: On the stability of the linear mapping in Banach spaces. Proc Am Math Soc. 72, 297-300 (1978)

19. Gajda, Z: On stability of additive mappings. Int J Math Math Sci. 14, 431-434 (1991)

20. Alotaibi, A, Mohiuddine, SA: On the stability of a cubic functional equation in random 2-normed spaces. Adv Diff Equ. 2012, 39 (2012)

21. Jun, KW, Kim, HM: The generalized Hyers-Ulam-Rassias stability of a cubic functional equation. J Math Anal Appl. 274 867-878 (2002)

22. Jung, SM, Kim, TS: A fixed point approach to the stability of the cubic functional equation. Bol Soc Mat Mexicana. 12(1):51-57 (2006)

23. Mohiuddine, SA: Stability of Jensen functional equation in intuitionistic fuzzy normed space. Chaos Solitons Fract. 42, 2989-2996 (2009)

24. Mohiuddine, SA, Şevli, H: Stability of Pexiderized quadratic functional equation in intuitionistic fuzzy normed space. J Comput Appl Math. 235, 2137-2146 (2011)

25. Mursaleen, M, Mohiuddine, SA: On stability of a cubic functional equation in intuition-istic fuzzy normed spaces. Chaos Solitons Fract. 42, 2997-3005 (2009)

26. Mirmostafaee, AK: A fixed point approach to almost quartic mappings in quasi fuzzy normed spaces. Fuzzy Sets Syst. 160, 1653-1662 (2009)

27. Radu, V: The fixed point alternative and the stability of functional equations. Sem Fixed Point Theory. 4(1):91-96 (2003)

28. Diaz, JB, Margolis, B: A fixed point theorem of the alternative for contractions on generalized complete metric space. Bull Am Math Soc. 126(74):305-309 (1968) 
29. Miheț, D, Radu, V: On the stability of the additive Cauchy functional equation in random normed spaces. J Math Anal Appl. 343, 567-572 (2008)

doi:10.1186/1687-1847-2012-48

Cite this article as: Mohiuddine and Alotaibi: Fuzzy stability of a cubic functional equation via fixed point technique. Advances in Difference Equations 2012 2012:48.

Submit your manuscript to a SpringerOpen ${ }^{\circ}$ journal and benefit from:

- Convenient online submission

- Rigorous peer review

- Immediate publication on acceptance

- Open access: articles freely available online

- High visibility within the field

- Retaining the copyright to your article

Submit your next manuscript at $\gg$ springeropen.com 